\title{
HEAT TRANSFER THROUGH METAL-INCLOSED INSULATION
}

\author{
By M.S. Van Dusen
}

ABSTRACT

The paper gives a mathematical treatment of the problem of heat transfer through an insulated metal-inclosed panel, such as a sheet-metal door, consisting essentially of a sheet-metal box filled with insulating material. The treatment is necessarily approximate, but the approximations involved are accurate enough for practical purposes. The effect of a solid metal thermal short circuit, such as a supporting member extending from one metal surface to the other, is also considered. Calculations and graphs are included, showing the relative magnitude of the heat transfer by metallic induction in a number of cases which might be considered in the design of this type of construction. It is shown that the increase in heat transfer through a metal-inclosed panel due to the presence of the metal may be as high as 100 per cent in many cases which might be considered in practical design.

\section{CONTENTS}

I. Introduction

II. Mathematical theory

III. Effect of a small thermal short circuit in the central portion of a panel

IV. Appendix I. Metal edges

V. Appendix II, Thermal short circuit 392

395

\section{INTRODUCTION}

The question of heat transfer through what might be termed an insulated panel, consisting of a slab of insulating material completely inclosed by sheet metal, the dimensions of the sides being large in comparison with the thickness of the panel, has been raised on a number of occasions, but the effect of metallic conduction around the edges of such a panel apparently has been a matter of conjecture. Insulated construction of this kind has many points in its favor, the principal one being that the insulating material is effectively sealed against moisture for a long period. The importance of this in refrigerator or cold-storage construction is well known. It is evident that definite information regarding the edge effect will be useful in the design of insulated construction of the metal-inclosed type. This paper offers a solution of the problem; necessarily approximate, but accurate enough for practical purposes.

\section{MATHEMATICAL THEORY}

It does not appear necessary to give the detailed mathematical analysis at this point, ${ }^{1}$ but it will be of interest to state the assumptions made and to indicate the general line of reasoning. Consider

1 Detailed analysis is given in the appendixes. 
a cross section (parallel to the general direction of heat flow) of an "insulated panel," together with adjacent ones, as shown by Figure 1.

The warm surface of the panel receives heat from the warmer air (at temperature $\theta_{2}$ ) in contact with it, part of which is conducted through the insulation and part through the metal around the edges. Since the thermal conductivity of the metal is much greater than that of the insulating material, the temperature at the point $A$ will be lower than that at $C$, and the temperature at $B$ will be higher than that at $D$. By symmetry, however, the temperature on the plane $E F$ will be the mean between $\theta_{2}$ and $\theta_{1}$, neglecting unimportant variations with temperature of thermal conductivity and surface resistance. Bearing this picture in mind, the following assumptions have been made:

1. Heat transfer between the warm air and the warm surface of the panel is proportional to the temperature difference between the warm air and the metal surface.

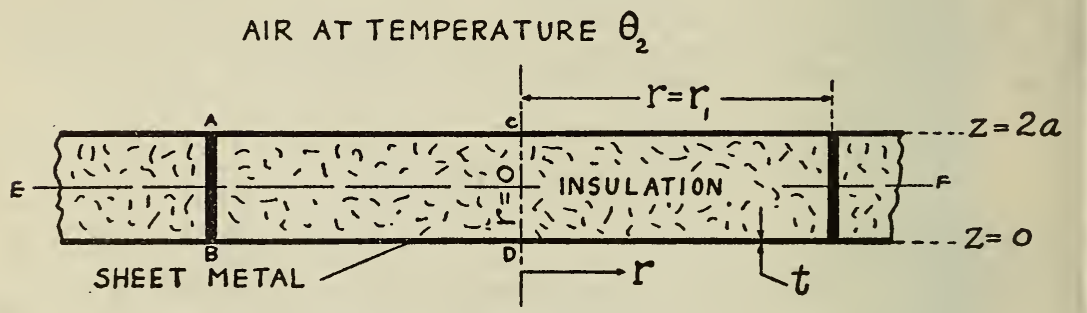

AIR AT TEMPER ATURE $\theta_{1}$

FIgURE 1.-Cross section of circular panel with complete metal inclosure

2. Heat transfer at any point between the warm metal surface and the insulation is proportional to the temperature difference between the metal at that point and the middle plane $E F$. Subsequent numerical calculations show that this approximation is accurate enough for practical purposes.

3. The sheet metal is relativ ely so thin and is such a good conductor compared to the insulation that temperature gradients in the metal perpendicular to its surface are negligible.

4. That portion of the metal between $A$ and $B$ suffers no net gain or loss of heat. This assumption is justified by considerations of symmetry.

5. Similar assumptions obviously apply to the cold metal surface.

It will be evident that the presence of the metal increases the heat transfer, but at the same time the heat transfer through the insulating material is less than it would have been were there no metal present. The net increase in heat transfer is the difference between these two effects.

Even with the simplifying assumptions enumerated above, the rigorous solution for a rectangular panel appears to be extremely complex. The solutions for a panel having a very large area so that corner effects can be neglected, and for a circular panel, however, are comparatively easy to obtain. In the range of practical cases the difference between these two solutions is so small that the uncertainty 
in their use for calculations applied to a rectangular panel is of no practical importance. The solution for a circular panel will be used in most of the numerical calculations, since it represents the closer approximation.

The following notation will be used:

$K=$ thermal conductivity of metal.

$t=$ thickness of metal.

$2 a=$ thickness of insulation.

$k=$ thermal conductivity of insulating material.

$h=$ surface conductance per unit area between air and metal.

$A=$ area of panel.

$P=$ perimeter of panel.

$I=$ net percentage increase in heat transfer due to presence of metal edges (basis, heat transfer without metal edges).

$T=$ transmittance of panel (total rate of heat transfer per unit temperature difference between air on both sides).

For a circular panel:

$$
I=\frac{P a h}{A k\left[\frac{a h+k}{K t}+\gamma \sqrt{\frac{a h+k}{a K t}}\right]}
$$

where

$$
\gamma=\frac{J_{0}\left(i \frac{2 A}{P} \sqrt{\frac{a h+k}{a K t}}\right)}{-i J_{1}\left(i \frac{2 A}{P} \sqrt{\frac{a h+k}{a K t}}\right)}
$$

The quantity $\gamma$ is a real function of the Bessel's functions $J_{0}$ and $J_{1}$ with pure imaginary arguments, and approaches unity with increasing size of panel. In the limit, therefore, equation (1) reduces to

$$
I=\frac{P a h}{A k\left[\frac{a h+k}{K t}+\sqrt{\frac{a h+k}{a K t}}\right]}
$$

Equation (3) can also be obtained by a direct method not involving the use of Bessel's cylindrical functions. ${ }^{3}$ The differences between equation (3) and equation (1) are only a few per cent for panels as large as 10 square feet in area.

If $h$ is very large, which amounts to saying that the temperature gradient in the metal edge is the same as that in the insulating material far removed from the edge, equation (3) reduces to:

$$
I=\frac{P K t}{A k}
$$

which could be written down directly from elementary principles, and represents the case in which the edge effect is greatest.

2 The derivation of this equation is given in Appendix I.

3 See Appendix I.

$118793^{\circ}-30-12$ 
If no metal were present around the edges, the transmittance $C$ of any panel would be:

$$
C=\frac{A k h}{2 a h+2 k}
$$

The transmittance $T$ of any panel with complete metal inclosure is evidently equal to $C(1+I)$.

From (5) and (1),

$$
T=\frac{A k h}{2 a h+2 k}\left[1+\frac{P a h}{A k\left[\frac{a h+k}{K t}+\gamma \sqrt{\frac{a h+k}{a K t}}\right]}\right]
$$

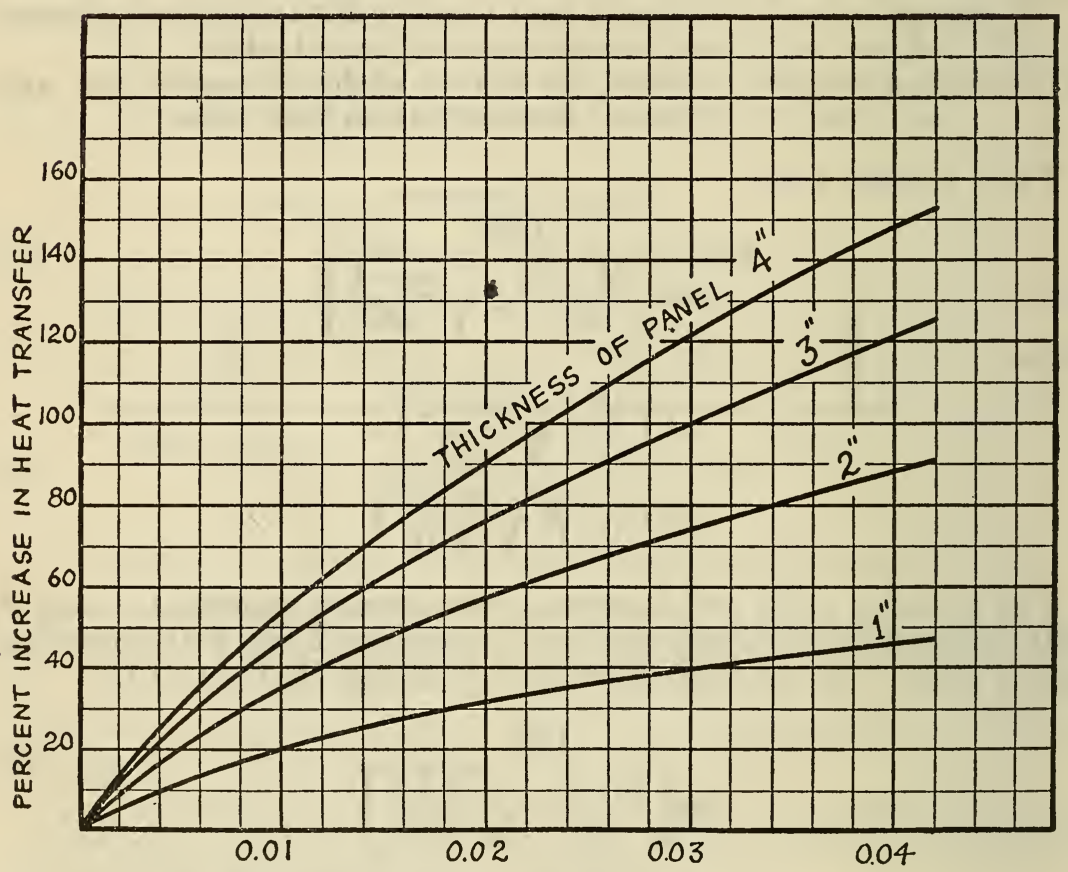

THICKNESS OF SHEET METAL - INCHES

FigURE 2.-Per cent increase in heat transfer through 40 by 40 inch panel, due to complete steel inclosure

Numerical calculations of practical cases show that the heat flow through the metal edges is not greatly affected even if the insulation is considered perfect. In such an event no assumption of linear heat flow through the insulating material is necessary, and it is therefore concluded that this assumption introduces no significant error in the calculation of heat flow in the metal. Numerical calculation also shows that the percentage decrease in heat transfer through the insulation due to the presence of the metal edges is small, and therefore the error involved in the assumption of linear heat flow through the insuiation is practically insignificant.

Figure 2 shows the percentage increase ( $I$, equation (1)) in heat transfer resulting from the use of a metal inclosure, plotted against the thickness of the metal (steel) used for the inclosure. A family of 
curves is shown, each member applying to a particular thickness of insulation. It will be noted that the percentage increase in heat transfer increases with thickness of insulation. The absolute increase in heat transfer, however, decreases with increasing thickness of insulation, as must be evident from elementary principles. Figure 3 shows the absolute values of the resistance and transmittance, plotted against thickness of insulation. Each curve corresponds to a particular thickness of metal.

In the calculations the following data have been used:

$k=0.002$ B. t. u. hour ${ }^{-1}$ inch $^{-1}\left({ }^{\circ} \mathrm{F} .\right)^{-1}$ (average insulating material). $h=0.01$ B. t. u. hour ${ }^{-1}$ inch $^{-2}\left({ }^{\circ} \mathrm{F} .\right)^{-1}$ (still air).

$K=2$ B. t. u. hour ${ }^{-1}$ inch $^{-1}\left({ }^{\circ} \mathrm{F} .\right)^{-1}$ (steel). $\frac{\mathrm{P}}{A}=0.1$ inch $^{-1}$ (40 by 40 inch panel).

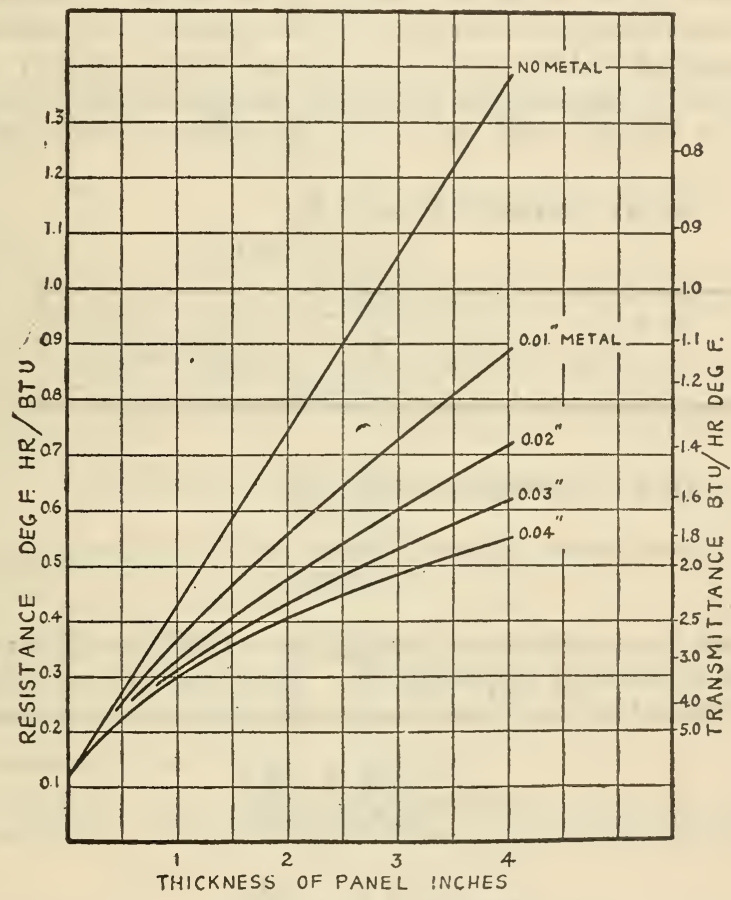

FIGURE 3.-Transmittance and resistance of 40 by 40 inch panel, as affected by the thickness of panel and thickness of steel inclosure

Since the percentage increase in heat transfer is nearly proportional to the value of $\frac{P}{A}$, values for panels of various sizes can readily be computed by a simple multiplication. To avoid inconsistencies, a single unit of length (the inch) has been used throughout.. ${ }^{4}$ Round

4 The familiar hybrid conductivity unit containing two units of length, the inch and the foot, is very inconvenient and illogical in dealing with heat flow in more than one direction. British units are used, since the majority of American engineers, to whom the paper is primarily addressed, are accustomed to think in terms of these units. The advantages of a decimal system are very grcat, but so many extraneous units; for example, the calorie, have been introduced into the various metric systems that the latter aro almost as bad as the various British systems. The universal use of decimal multiples of e. g. s. units would be a great step forward. 
numbers, closely approximating average experimental values, have been used for the thermal properties of the materials. For relatively small changes in the value of $k$ (through the range of ordinary insulating materials) the percentage increase in heat transfer is approximately inversely proportional to $k .^{5}$ The effect of changes in the value of $h$ can not be approximated so simply, and recourse must be made to the original equations. The value of the quantity $\gamma^{6}$ varies little in the range of practical cases, and the average value of 1.05 is sufficiently close. In the calculations for Figure $2, \gamma$ varied from 1.03 to 1.07 .

\section{EFFECT OF A SMALL THERMAL SHORT CIRCUIT IN THE CENTRAL PORTION OF A PANEL}

In Figure $4, R$ is a rod of radius $r_{2}$ in metallic thermal contact with the sheet metal on both sides of the panel. This thermal short circuit is assumed to have the same thermal conductivity as the sheet metal, and to be far enough removed (a foot or so) from the edges or from other similar rods such that the effects of the latter on the

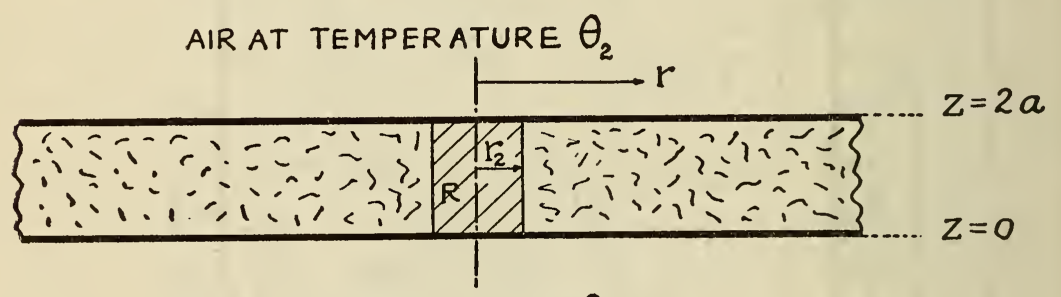

\section{AIR AT TEMPERATURE $\theta_{1}$}

FIgURe 4.-Cross section of metal covered panel with metal short circuiting connection

particular rod in question are negligible. Outline of the solution of this problem is given in Appendix II. If the rate of heat flow through the rod is denoted by $\mathrm{H}_{2}$, then for rod diameters up to about one-half inch,

where

$$
H_{2}=\frac{\pi r_{2}^{2} h\left(\theta_{2}-\theta_{1}\right)}{2 a t \alpha^{2}+\lambda \alpha r_{2}}
$$

and

$$
\lambda=2.3 \alpha r_{2} \log _{10} \frac{1.12}{\alpha r_{2}}
$$

$$
\alpha^{2}=\frac{a h+k}{a K t}
$$

The effect of the presence of the metal connection on heat flow through the insulation is not significant, since the area of the insula-

T 5 The approximation becomes less exact as the thickness of the insulation is decreased.

6 Values of $\gamma$ for values of the argument greater than 4 (always the case in a panel of practical dimensions) can readily be computed by means of the very good approximation

$$
\gamma=\frac{J_{0}(i x)}{-i J_{1}(i x)}=\sqrt{\frac{x}{x-1}}
$$

This equation can be deduced from the semiconvergent expressions for Bessel's functions. 
tion is very large compared with that of the disturbed region around the metal connection.

The rate of heat flow through a steel short circuit is plotted in Figure 5. The figure is self-explanatory and need not be discussed io any extent. By comparing the values with those in Figure 3, $t$ will be noticed that the transmittance of a short circuit of reasonable ize is small compared to that of a 40 by 40 inch panel.

If the short-circuiting rod is not in metallic thermal contact with he metal surfaces, or if no metal surfaces are present, its effect is ractically insignificant on account of its relatively small area. A

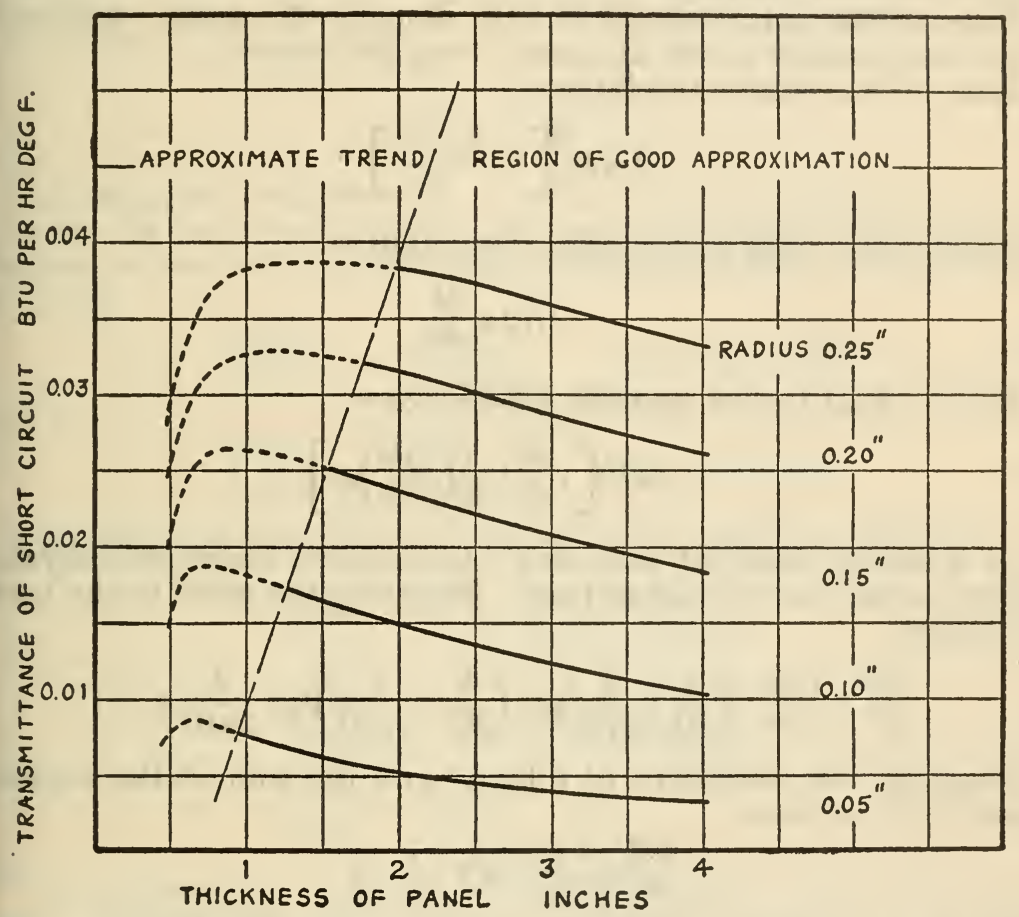

FIGURE 5.-Effective transmittance of thermal short circuiting steel connection, as affected by thickness of panel and size of metal connection

Sheet steel on surfaces 0.02 inch thick.

good example of this is a nail running completely through the insulation. In such a case the heat flow is evidently given by

$$
H_{2}=\frac{\pi r_{2}^{2}\left(\theta_{2}-\theta_{1}\right) h}{2}
$$

the thermal resistance of the rod itself being negligible in comparison with the surface resistances on each end. ${ }^{7}$

For a discussion of the effect of metallic members projecting part way into the insulating material, see F. H. Schofield, Phil. Mag., 7 series, 5, p. 567; 1928. 


\section{APPENDIX I. METAL EDGES}

Figure 1 shows a cross section of a circular panel surrounded at the edges in such a way that no heat is lost or gained laterally. Tht general outline of the procedure and the assumptions used in the derivation of the equations has already been given and will not $b$ repeated here.

Consider the state of affairs in the metal on the warm side $z=2 a$ with particular reference to any ring element of infinitesimally smal width $\delta r$. It is evident from symmetry that the temperature is uniform around the ring.

Rate of heat gain from air at $\theta_{2}$ to element $=2 \pi r \delta r h\left(\theta_{2}-\theta\right)$ where is the temperature at the distance $r$ from the center.

Rate of heat loss to insulation $=$

$$
2 \pi r \delta r \frac{k}{a}\left[\theta-\frac{\theta_{2}+\theta_{1}}{2}\right] \text {. }
$$

Rate of heat gain by metallic conduction $=$

$$
-K t 2 \pi r \frac{d \theta}{d r}
$$

Rate of heat loss by metallic conduction $=$

$$
-2 \pi K t\left[r \frac{d \theta}{d r}+\frac{d}{d r}\left(r \frac{d \theta}{d r}\right) \delta r\right]
$$

In a steady state of heat flow the element under consideration suffers no net gain or loss of heat. Equating the gains to the losses we obtain

$$
\frac{d^{2} \theta}{d r^{2}}+\frac{1}{r} \frac{d \theta}{d r}-\left(\frac{h}{K t}+\frac{k}{a K t}\right) \theta+\left(\frac{h}{K t}+\frac{k}{2 a K t}\right) \theta_{2}+\frac{k}{2 a K t} \theta_{1}=0
$$

Denoting the coefficient of $\theta$ by $\alpha^{2}$ and the sum of the constant terms by $b$, we have

$$
\frac{d^{2} \theta}{d r^{2}}+\frac{1}{r} \frac{d \theta}{d r}-\alpha^{2} \theta+b=0
$$

This is a modified form of Bessel's equation of order zero, and particular solution is easily found to be

$$
\theta=A J_{0}(i \alpha r)+\frac{b}{\alpha^{2}}
$$

where $A$ is an arbitrary constant, $J_{0}$ denotes the Bessel function o zero order, and $i$ is the usual symbol for $\sqrt{-1}$. The function $J_{0} i$ a real function of the pure imaginary argument $i \alpha r$.

The complete solution of equation (2) will contain a Bessel's func tion of the second kind which becomes infinite at the origin, and can not, therefore, appear in the solution of the present problem. If $\theta$ denotes the temperature (as yet unknown) of the edge $r=r_{1}$ and $z=2 a$, then equation (3) becomes

$$
\theta=\left(\theta_{3}-\frac{b}{\alpha^{2}}\right) \frac{J_{0}(i \alpha r)}{J_{0}\left(i \alpha r_{1}\right)}+\frac{b}{\alpha^{2}}
$$


On the cold surface $z=0$ a similar argument yields the equation

$$
\theta=\left(\theta_{4}-\frac{c}{\alpha^{2}}\right) \frac{J_{0}(i \alpha r)}{J_{0}\left(i \alpha r_{1}\right)}+\frac{c}{\alpha^{2}}
$$

where $\theta_{4}$ is the temperature on the cold edge $r=r_{1}$ and $z=0$, and

$$
c=\left(\frac{h}{K t}+\frac{k}{2 a K t}\right) \theta_{1}+\frac{k}{2 a K t} \theta_{2}
$$

Since no heat is lost or gained by the metal along the edge, the temperature distribution in the metal at the edges must be linear along the $z$ axis. In terms of the unknown temperatures $\theta_{3}$ and $\theta_{4}$, this distribution is given by

$$
\theta=\theta_{4}+\frac{\theta_{3}-\theta_{4}}{2 a} z
$$

The unknown temperatures $\theta_{3}$ and $\theta_{4}$ can be evaluated by considering the fact that there can be no discontinuity in temperature yradient in the metal where it bends around the edges. We therefore san write

and

$$
\left(\frac{\partial \theta}{\partial r}\right)_{r=r_{1}}=-\left(\frac{\partial \theta}{\partial z}\right)_{z=2 a} \quad \text { (on warm side) }
$$

$$
\left(\frac{\partial \theta}{\partial r}\right)_{r=r_{1}}=\left(\frac{\partial \theta}{\partial z}\right)_{z=0} \quad \text { (on cold side) }
$$

Differentiating equations (4), (5), and (7), yields, respectively:

$$
\begin{aligned}
& \left(\frac{\partial \theta}{\partial r}\right)_{r_{1}}=-\left(\theta_{3}-\frac{b}{\alpha^{2}}\right) \alpha \frac{i J_{1}\left(i \alpha r_{1}\right)}{J_{0}\left(i \alpha r_{1}\right)} \\
& \left(\frac{\partial \theta}{\partial r}\right)_{r_{1}}=-\left(\theta_{4}-\frac{c}{\alpha^{2}}\right) \alpha \frac{i J_{1}\left(i \alpha r_{1}\right)}{J_{0}\left(i \alpha r_{1}\right)} \\
& \left(\frac{\partial \theta}{\partial z}\right)=\frac{\theta_{3}-\theta_{4}}{2 a}
\end{aligned}
$$

The values of $\theta_{3}$ and $\theta_{4}$ can be obtained by the use of these equaions. Substituting their values in equations (4) and (5). and definng a quantity,

ve have finally

$$
\gamma=\frac{J_{0}\left(i \alpha r_{1}\right)}{-i J_{1}\left(i \alpha r_{1}\right)}
$$

$$
\begin{array}{ll}
\theta=\frac{b}{\alpha^{2}}-\frac{\gamma(b-c)}{2 \alpha^{2}(\gamma+a \alpha)} \frac{J_{0}(i \alpha r)}{J_{0}\left(i \alpha r_{1}\right)} & \text { (warm side) } \\
\theta=\frac{c}{\alpha^{2}}+\frac{\gamma(b-c)}{2 \alpha^{2}(\gamma+a \alpha)} \frac{J_{0}(i \alpha r)}{J_{0}\left(i \alpha r_{1}\right)} & \text { (cold side) }
\end{array}
$$

The temperature difference $\Delta \theta$ between corresponding points at $=2 a$ and $z=0$ on a cylinder of radius $r$ is the difference between quations (13) and (14), or

$$
\Delta \theta=\frac{b-c}{\alpha^{2}}-\frac{\gamma(b-c)}{\alpha^{2}(\gamma+a \alpha)} \frac{J_{0}(i \alpha r)}{J_{0}\left(i \alpha r_{1}\right)}
$$


The rate of heat transfer through the insulation (on the original assumption of linear flow) is given by

$$
H_{1}=\int_{0}^{r_{1}} 2 \pi r \frac{k}{2 a} \Delta \theta d r
$$

Substituting equation (15) in equation (16) and integrating, yields

$$
H_{1}=\frac{\pi k(b-c) r_{1}^{2}}{2 a \alpha^{2}}\left[1-\frac{2}{r_{1} \alpha(\gamma+a \alpha)}\right]
$$

If no metal were present around the edges, a very elementary calculation shows that the rate of heat transfer would be equal to the coefficient of the bracketed expression in equation (17). The decrease in heat flow through the insulation, due to the presence of the metal around the edges, is therefore given by

$$
\frac{\pi k(b-c) r_{1}}{a \alpha^{3}(\gamma+a \alpha)}
$$

The heat flow through the metal edge is evidently given by the expression:

$$
\frac{2 \pi r_{1} K t\left(\theta_{3}-\theta_{4}\right)}{2 a}
$$

By the use of equations (13) and (14), this expression is easily evaluated and found to be:

$$
\frac{\pi r_{1} K t(b-c)}{\alpha(\gamma+a \alpha)}
$$

The net increase $\Delta H$ in total rate of heat flow through the panel, due to the presence of the metal edges, is the difference between equation (20) and (18), or

$$
\Delta H=\frac{\pi r_{1}(b-c)}{\alpha(\gamma+a \alpha)}\left[K t-\frac{k}{a \alpha^{2}}\right]
$$

Were there no metal present around the edges, the total rate of heat transfer $H$ through the panel would be

$$
H=\frac{\pi r_{1}^{2} h k\left(\theta_{2}-\theta_{1}\right)}{2(a h+k)}
$$

The percentage increase in heat transfer, due to the presence of the metal edges, is equal to $\frac{\Delta H}{H}$. Substituting the values of $b, c$, and $\alpha$, we have finally,

$$
\frac{\Delta H}{H}=\frac{2 a h}{k r_{1}\left(\frac{a h+k}{K t}+\gamma \sqrt{\frac{a h+k}{a K t}}\right)}
$$

This is identical with equation (1) in the text, since $\frac{2}{r_{1}}=\frac{P}{A}$. 
A similar analysis applied to an infinite straightedge (therefore assuming at the outset that the heat flow in the metal edge is directly proportional to the length of the edge and independent of the area of the panel) involves the use of exponential functions and yields equation (23) in the special limiting case where $\gamma \rightarrow 1$. Evidently this must necessarily follow, and amounts to nothing more than a check on the analysis.

\section{APPENDIX II, THERMAL SHORT CIRCUIT}

Referring to Figure 4, the differential equation of heat fiow in the metal surfaces in the range $r>r_{2}$ is evidently the same as in the previous case. The particular solution required, however, is different, since the boundary conditions are different. We are no longer concerned with the value of $\theta$ when $r=0$, but when the value of $r$ becomes large, $\theta$ must approach the value it would assume were there no metal short circuits. The general solution of equation (2), Appendix I is

$$
\theta=A^{\prime} J_{0}(i \alpha r)+B^{\prime} Y_{0}(i \alpha r)+\frac{b}{\alpha^{2}}
$$

By choosing certain values of the arbitrary constants $A^{\prime}$ and $B^{\prime}$, we can write

$$
\theta=A\left\{\left[\log \frac{2 i}{\beta}\right][J(i \alpha r)]-Y_{0}(i \alpha r)\right\}+\frac{b}{\alpha^{2}}
$$

The quantity in the braces is real, vanishes for infinite values of $r,{ }^{8}$ and is therefore the solution required, since $\frac{b}{\alpha^{2}}$ is the temperature of the metal surface were there no metal short circuits present.

$Y$ is the more common notation for Bessel's functions of the second kind, although many other notations are used. $\beta$ is a fixed numerical constant having the value 1.7811. The bracketed expression in equation (2) is a real function of $r$ and was denoted by Hankel ${ }^{9}$ as

$$
\frac{i \pi}{2} H_{0}(i \alpha r)
$$

When

$$
r=r_{2} \text { and } z=2 a, \theta=\theta_{3} .
$$

Eliminating the constant $A$ from equation (2) by this means yields

$$
\theta=\frac{b}{\alpha^{2}}+\left(\theta_{3}-\frac{b}{\alpha^{2}}\right) \frac{H_{0}(i \alpha r)}{H_{0}\left(i \alpha r_{2}\right)}
$$

valid in the range $r>r_{2}$ and $2 a-t<z<2 a$.

Within the cylindrical rod short circuiting the two thin metal surfaces, we must satisfy the equation $\nabla^{2} \theta=0$ with the following boundary conditions: When

$$
z=a, \theta=\frac{\theta_{2}+\theta_{1}}{2}
$$

8 See Jahnke-Emde "Funktionentafeln mit Formeln und Kurven," Teubner, p. 94; 1909.

- Hankel, Mathematische Annalen, 1, p. 483; 1869; 8, p. 453; 1875. 
$z=2 a, \frac{\partial \theta}{\partial z}=0$

(neglecting the very small amount of heat received directly from the warm air over the relatively small area $\pi r_{2}^{2}$ ).

$$
\begin{gathered}
\left.\underset{r=r_{2}}{2 a-t<z<2 a}\right\}, \frac{\partial \theta}{\partial r}=\left(\frac{d \theta}{d r}\right)_{r=r_{2}} \text { from equation (3) } \\
\left.\begin{array}{c}
a<z<2 a-t \\
r=r_{2}
\end{array}\right\}, \frac{\partial \theta}{\partial r}=0
\end{gathered} .
$$

The solution satisfying these conditions, valid in the range $r<r_{2}$ and $a<z<2 a$, can be found without great difficulty, and is as follows:

where

$$
\begin{gathered}
\theta=\frac{\theta_{2}+\theta_{1}}{2}-\frac{8 a \alpha\left(\frac{b}{\alpha^{2}}-\theta_{3}\right)}{\lambda \pi^{2}} \sum_{n=1}^{n=\infty} \frac{\sin \frac{(2 n-1) \pi}{2} \sin \frac{(2 n-1) \pi t}{2 a}}{(2 n-1)^{2} i J_{1}\left(i \frac{(2 n-1) \pi r_{2}}{2 a}\right)} \\
\sin \frac{(2 n-1) \pi(z-a)}{2 a} J_{0}\left(i \frac{(2 n-1) \pi r}{2 a}\right)
\end{gathered}
$$

$$
\lambda=\frac{H_{0}\left(i \alpha r_{2}\right)}{i H_{1}\left(i \alpha r_{2}\right)}
$$

At the boundary region $r=r_{2}$ and $2 a-t<z<2 a$, equations ( 3 ) and (4) should give identical values of temperature $\left(\theta_{3}\right)$ as well as normal derivative, in order to satisfy the physical demand of continuity of temperature. Evidently they will not do this exactly, but the discrepancy is negligible, since over the small distance from $z=2 a-t$ to $z=2 a$, the temperature given by equation (4) is very nearly constant. The elimination of $\theta_{3}$ by using equations (3) and (4) is therefore accurate enough for the present purpose.

When $r=r_{2}$ and $z=2 a$

$$
\theta=\theta_{3}=\frac{\theta_{2}+\theta_{1}}{2}-\frac{8 a \alpha\left(\frac{b}{\alpha^{2}}-\theta_{3}\right)}{\lambda \pi^{2}} \sum_{n=1}^{n=\infty} \frac{\sin \frac{(2 n-1) \pi t}{2 a}}{(2 n-1)^{2}} \frac{J_{0}\left(i \frac{(2 n-1) \pi r_{2}}{2 a}\right)}{i J_{1}\left(i \frac{(2 n-1) \pi r_{2}}{2 a}\right)}
$$

Solving for $\theta_{3}$

$$
\theta_{3}=\frac{\frac{8 a b}{\pi^{2} \alpha \lambda} \sum+\frac{\theta_{2}+\theta_{1}}{2}}{1+\frac{8 a \alpha}{\pi^{2} \lambda} \sum}
$$

The rate of heat flow, $\mathrm{H}_{2}$, down the metal rod is evidently equal to

$$
2 \pi r_{2} K t \frac{d \theta}{d r} \quad \text { (from equation (3)) }
$$

therefore,

$$
H_{2}=\frac{2 \pi r_{2} K t \alpha}{\lambda}\left(\frac{b}{\alpha^{2}}-\theta_{3}\right)
$$


Substituting the value of $\theta_{3}$, we have

$$
H_{2}=\frac{2 \pi r_{2} K t \alpha}{\lambda}\left[\frac{b}{\alpha^{2}}-\frac{\frac{\theta_{2}+\theta_{1}}{2}+\frac{8 a b}{\pi^{2} \alpha \lambda} \sum}{1+\frac{8 a \alpha}{\pi^{2} \lambda} \sum}\right]
$$

The series, denoted by the abbreviation $\Sigma$, is approximately equal, for small values of $r_{2}$ less than about 0.25 inch, to the expression

$$
\sum=\frac{\pi^{2} t}{4 r_{2}}
$$

This equation is obtained by considering the limiting forms of expression of the quantities involved as $r_{2}$ approaches zero. The limitations of the approximation can be shown only by numerical calculation. The approximate value of $\lambda$ (equation (9) in text) is at least as accurate as the approximation for $\Sigma$, and was obtained by the same method.

Using this approximation, and substituting the value of the quantity $b$, we have finally, after considerable algebra,

$$
H_{2}=\frac{\pi r_{2}{ }^{2} h\left(\theta_{2}-\theta_{1}\right)}{2 a t \alpha^{2}+r_{2} \alpha \lambda}
$$

This expression can be obtained directly by assuming at the outset that each end of the metal short-circuiting post has an infinitely conducting surface layer of small thickness $t$.

Washington, April 30, 1930. 